\title{
A Roadmap for Designing an Automated Visual Inspection System
}

\author{
D.Palani Kumar, \\ Kamaraj College of Engineering \& \\ Technology, \\ Post Box No. 120, SPGC Nagar, \\ Virudhunagar - 626 001, India
}

\author{
K. Kannan, \\ Kamaraj College of Engineering \& \\ Technology, \\ Post Box No. 120, SPGC Nagar, \\ Virudhunagar - 626 001, India
}

\begin{abstract}
The new machine vision that is emerging is already generating millions of dollars per year in thousands of successful applications. Machine vision is becoming established as a successful tool for industrial automation where $100 \%$ inspection of manufactured parts during production is becoming a reality. Two-thirds of applications of machine vision are found in quality control in which objects are categorized using its dimensions in metric measurements that are extracted from its 2D-pixel image. Building a machine vision system requires careful selection of appropriate sensor, lens, extraction and integration of information from the available cues, sensed data and evaluation of system performance and robustness. In this paper, it is proposed to provide a roadmap with an overview of Imaging system, Radiometric and Geometric Modeling to design a machine vision system with the illustration of design of automated visual inspection system to image new bottles on a conveyor line at a distance of $100 \mathrm{~cm}$ from the camera in order to ensure $30 \mathrm{~mm}$ diameter of the bottle neck.
\end{abstract}

\section{Keywords}

Automated visual Inspection System, Machine Vision, Imaging System, CCD Sensors, Frame grabber.

\section{INTRODUCTION}

The driving forces in today's manufacturing environments are: quality improvement, cost reduction, Increased volume, and shorter cycle times for manufacturing [4]. Improved quality is a key factor in satisfying the demands on modern manufacturing. The quality of many raw materials, parts, and products can be measured by electrical or mechanical means, or by visual inspection. To produce the good quality products, it is necessary to inspect each and every part that comprises the product. Inspection by eye is costly, subjective, qualitative, inaccurate, eye-straining, and timeconsuming. For high speed and real time applications, manual inspection is not possible. Therefore, more and more manufacturers look for fast, accurate, reliable, and consistent automated visual inspection of their products.

During the last two decades, machine vision systems, which perform automated visual inspection, have been applied slowly but surely to a variety of manufacturing challenges, all with the goal of improving quality and productivity in the manufacturing processes $[4,5,6,7,8]$. Machine Vision is defined as acquisition of the image of an object of interest followed by processing and interpretation of this image using computer for some useful applications like industrial inspection. Machine vision unifies illumination, imaging,

image processing and analysis, and material handling technologies to provide noncontact localisation, characterization, and manipulation of stationary or moving objects. Features such as size, shape, colour, etc. are used to discriminate between good and defected objects. For real-time operation, the inspection process must be able to keep up with the manufacturing process. This is often the most challenging task when designing a machine vision system, as objects may be produced at high rates [8]. Fortunately, machine vision systems often operate in constrained and controlled environments in which there is control over illumination, image contrast and resolution, position and orientation of objects, or knowledge about the objects to be inspected.

Our expectation from a visual system is to perform the following operations: the image acquisition and analysis, the recognition of certain features or objects within that image, and the exploitation and imposition of environmental constraints [10].

Scene constraint is the first consideration for the machine vision system. The situation of the scene must be recognized by the machine vision designer and according to the required application such a machine should be developed. The hardware for this sub-system consists of the light source for the active imaging, and required optical systems. Different lighting techniques such as the structured lighting can be used for such purpose. The process of vision system starts with the image acquiring in which representation of the image data, image sensing and digitization is accomplished. Image sensing is the next step in order to obtain a proper image from the illuminated scene. Digitization is the next process in which image capturing and image display are accomplished. The last step in this process is the image processing in which a more suitable image is prepared.

In this paper, we describe a machine vision system that uses an Imaging system consisting of imaging sensor to acquire the image of object of interest. Even though more number of sensors is available for capturing the image, the most popular sensor for machine vision applications is the Charge Coupled Device (CCD) paired with frame grabber. In quality control using machine vision system, objects are extracted from its 2D image formed using the amount of light energy received and directed by the set of optical glasses called lens onto the photo sensitive elements[1]. Hence, a machine vision system has to be designed by properly selecting the appropriate sensor, lens, extraction and integration of information. 


\section{OVERVIEW OF IMAGING SYSTEM}

The following section presents the overview of the imaging system, which is used to acquire image of object of interest for any image processing applications.

\subsection{CCD Camera}

CCD Cameras are the most common imaging sensors used in machine vision applications due to its small size (1/4", 1/3", 1/2", $2 / 3$ " and 1"), lightweight, impressive dynamic range and linear relationship between incoming energy and outgoing signal. The incoming light from the scene is collected by the set of optical glasses called lens and directed onto the photosensitive elements (SELs) of the camera. All sels have the same geometry, organized in a rectangular array and isolated electrically from their neighbors by a channel stop. When photon strikes a silicon atom resting in or near a sel, it will produce a free electron and a hole created by the temporary absence of the electron. The free electron is then collected at each site in a potential well, while the hole is forced away from the well. The amount of electrons generated depends on the incoming photon flux, exposure period and the physical properties of the sels. After the charge packets are collected, they have to be transferred to the output stage where they are amplified and converted into voltage.

\subsection{Frame grabber}

Frame grabber is the interface between a camera and a computer and works with the CCD simultaneously. It reads the analog signal in composite video format. This format consists of image content periods corresponding to the content of a single line, separated by horizontal synchronization pulses, HSYNC. The transmission of the image from the camera to the frame grabber is in fields. The fields are separated by the vertical synchronization pulses, VSYNC. The frame grabber samples the analog signal at a sampling frequency set by its analog to digital converter. The sampled voltage levels are converted to integer gray values.

\section{RADIOMETRIC MODEL OF IMAGING SYSTEM}

The following section presents radiometric model of the imaging system, the transfer of the signal from the scene through the detector, the on-chip amplifier, the off-chip amplifier and A/D converter. An imaging sensor may produce value I (number of electrons) at a collection site is given by $\mathrm{I}=T \int_{\lambda} \int_{\mathrm{Y}} \int_{x} B(x, y, \lambda) S_{r}(x, y) q(\lambda) d x d y d \lambda$

where $\mathrm{T}$ is integration time in seconds, $(\mathrm{x}, \mathrm{y})$ are continuous coordinates in sensor plane, $\mathrm{B}(\mathrm{x}, \mathrm{y}, \lambda)$ is the incident spectral irradiance, $\mathrm{S}_{\mathrm{r}}(\mathrm{x}, \mathrm{y})$ is the spectral response of the collection site, and $q(\lambda)$ is the ratio of electrons to the incident light. After that the electrons are converted into signals and amplified using on-chip and off-chip amplifiers. The signal from the camera is measurable and calculated using the formulae,

$\mathrm{V}_{\text {camera }}=\mathrm{N}_{\mathrm{e}} \mathrm{GG}_{1} \mathrm{q} / \mathrm{C}$

where $q$ is the electron charge, $G$ is the gain of the on-chip amplifier, $G_{1}$ is the gain of the off-chip amplifier, $N_{e}$ is the number of electrons in the charge packet and $\mathrm{C}$ is the capacitance of the output node[2].

\section{GEOMETRIC MODEL OF IMAGING SYSTEM}

Let $\mathrm{P}\left(\mathrm{X}_{\mathrm{w}}, \mathrm{Y}_{\mathrm{w}}, \mathrm{Z}_{\mathrm{w}}\right.$ be the $3 \mathrm{D}$ co-ordinates of an object point ' $\mathrm{P}$ ' in the world co-ordinate system and $\mathrm{P}\left(\mathrm{X}_{\mathrm{c}}, \mathrm{Y}_{\mathrm{c}}, \mathrm{Z}_{\mathrm{c}}\right)$ be the $3 \mathrm{D}$ co-ordinates of the same point ' $\mathrm{P}$ ' in the $3 \mathrm{D}$ camera coordinate system, which is centered at the point ' $\mathrm{O}_{\mathrm{c}}$ ' the optical center, with the $\mathrm{Zc}$ axis, the same as optical axis. Refer figure 1. $\left(\mathrm{X}_{\mathrm{i}}, \mathrm{Y}_{\mathrm{i}}\right)$ is the image co-ordinate system centered at ' $\mathrm{O}_{\mathrm{i}}$ ' and parallel to $X_{c}$ and $Y_{c}$ axis. The effective focal length ' $F$ ' is the distance between the front image plane and the optical center. $P\left(X_{u}, Y_{u}\right)$ is the image co-ordinates of $P\left(X_{c}, Y_{c}, Z_{c}\right)$ if a perfect pin hole camera is used. $P\left(X_{d}, Y_{d}\right)$ are the actual image co-ordinates which differs from $\mathrm{P}\left(\mathrm{X}_{\mathrm{u}}, \mathrm{Y}_{\mathrm{u}}\right)$ due to lens distortion. The overall transformation from $\left(X_{w}, Y_{w}, Z_{w}\right)$ to $\left(X_{f}\right.$, $\mathrm{Y}_{\mathrm{f}}$ ) can be subdivided into four steps[1,2].

Step 1: Rigid body transformation from the object world coordinate system $\left(\mathrm{X}_{\mathrm{w}}, \mathrm{Y}_{\mathrm{w}}, \mathrm{Z}_{\mathrm{w}}\right)$ to the camera $3 \mathrm{D}$ co-ordinate system $\left(X_{c}, Y_{c}, Z_{c}\right)$ is given by

$$
\left[\begin{array}{llll}
X_{\mathrm{c}} & Y_{\mathrm{c}} & \mathrm{Z}_{\mathrm{c}} & 1
\end{array}\right]^{\mathrm{T}}=[\mathrm{R}]\left[\begin{array}{llll}
\mathrm{X}_{\mathrm{w}} & \mathrm{Y}_{\mathrm{w}} & \mathrm{Z}_{\mathrm{w}} & 1
\end{array}\right]^{\mathrm{T}}+[\mathrm{T}]
$$

where $[\mathrm{R}]$ is the $3 \mathrm{X} 3$ Rotation Matrix and $[\mathrm{T}]$ is the Translation vector.

Step 2: Transformation from $3 \mathrm{D}$ camera co-ordinate $\left(\mathrm{X}_{\mathrm{c}}, \mathrm{Y}_{\mathrm{c}}\right.$, $\left.Z_{c}\right)$ to ideal undistorted image co-ordinate $\left(X_{u}, Y_{u}\right)$ using perspective projection is governed by

$$
\begin{aligned}
& \mathrm{Xu}=\mathrm{F}\left(\frac{r_{1} X w+r_{2} Y w+r_{3} Z w+T x}{r_{7} X w+r_{8} Y w+r_{9} Z w+T z}\right) \\
& \mathrm{Yu}=\mathrm{F}\left(\frac{r_{4} X w+r_{5} Y w+r_{6} Z w+T y}{r_{7} X w+r_{8} Y w+r_{9} Z w+T z}\right)
\end{aligned}
$$

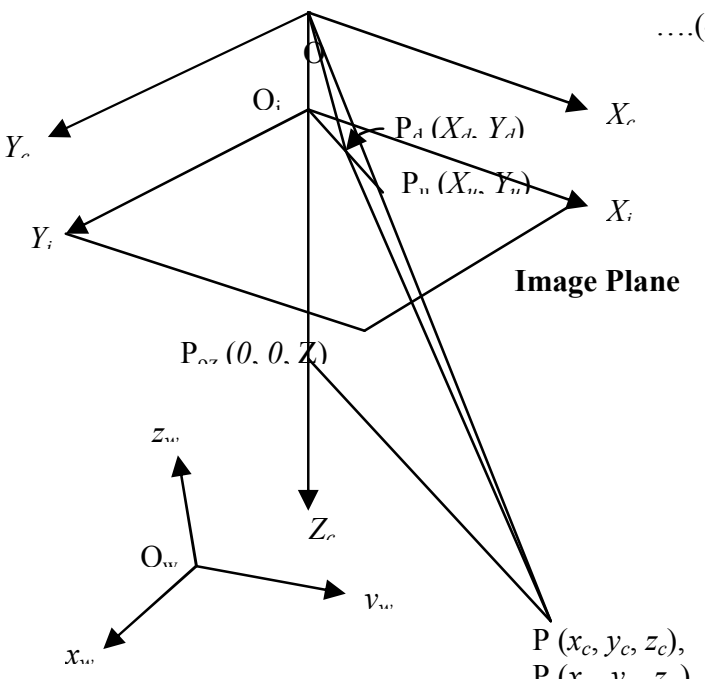

Figure 1: Geometric Camera Model

Step 3 : Undistorted Image co-ordinates $\left(\mathrm{X}_{\mathrm{u}}, \mathrm{Y}_{\mathrm{u}}\right)$ to distorted image co-ordinate considering only radial lens distortion is expressed as

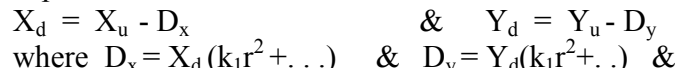


$\mathrm{r}^{2}=X_{d}^{2}+Y_{d}^{2}$

Step 4 : Distorted Image Co-ordinate $\left(\mathrm{X}_{\mathrm{d}}, \mathrm{Y}_{\mathrm{d}}\right)$ to computer image co-ordinate $\left(\mathrm{X}_{\mathrm{f}} \mathrm{Y}_{\mathrm{Y}}\right)$

$\mathrm{X}_{\mathrm{F}}=\left(\mathrm{F}_{\mathrm{c}} / \mathrm{F}_{\mathrm{f}}\right)\left(\mathrm{X}_{\mathrm{d}} * \mathrm{~N}_{\mathrm{fx}} / \mathrm{dx} * \mathrm{~N}_{\mathrm{cx}}\right)+\mathrm{C}_{\mathrm{x}}$.

$\mathrm{Y}_{\mathrm{F}}=\left(\mathrm{Y}_{\mathrm{d}}+\mathrm{dy}\right)+\mathrm{C}_{\mathrm{y}}$

where $\left(C_{x}, C_{y}\right)$-Center of computer image coordinates; $d_{x} \& d_{y}$ Center to center distance between pixels in $\mathrm{X} \& \mathrm{Y}$ direction ; $\mathrm{N}_{\mathrm{cx}}-$ Number of sels; $\mathrm{N}_{\mathrm{fx}}$-Number of pixels; $\mathrm{F}_{\mathrm{c}} \& \mathrm{~F}_{\mathrm{f}}$ - Frequency of the camera \& $\mathrm{A} / \mathrm{D}$ converter.

\section{DESIGN METHODOLOGY}

A system is to be designed to image new bottles on a conveyer line to ensure the bottle's neck as $30 \mathrm{~mm}$ diameter. Some of the important specifications are as follows: Bottle diameter is $100 \mathrm{~mm}$ in diameter, Throughput is 40 bottles per second, Conveyor speed is $800 \mathrm{~mm}$ per second and Working distance is $1000 \mathrm{~mm}$ with an accuracy of $0.1 \mathrm{~mm}$ position measurement. The various steps in the initial design, which contribute towards obtaining an image, are as follows:

\subsection{Selection of camera:}

The nature of this application requires an imaging sensor with an excellent noise performance, much better than for video applications. CCDs are superior to Complementary Metal Oxide Semiconductor (CMOS) image sensors, as far as SNR and dynamic range are concerned. So, CCD image sensor is selected for this application.

\subsection{Choosing camera view:}

Choosing the camera view requires the information about the feature of interest, where they are located on the part and pose of the part. For this application, the camera view is selected perpendicular to the direction of motion, bottles are moved horizontally in the conveyor line and bottle necks are facing the camera.

\subsection{Evaluation of resolution:}

There are three types of resolutions and they are

- Image resolution which is defined as the number of rows and columns of pixels in the image. Assuming sub-pixel resolution can be used and that an accuracy of one pixel can be achieved, the needed resolution is $100 \mathrm{~mm} / 0.1 \mathrm{~mm}=$ 1000 pixels. For this image resolution, a high resolution CCD camera JAI CV M1 progressive scan CCD camera with the resolution of 1300 X 1030 should work.

- Spatial resolution which is defined as the spacing between pixel centers when they are mapped onto the scene. For the JAI CV M1 CCD camera, the spatial resolution is $0.0067 \mathrm{~mm}$ $\mathrm{X} 0.0067 \mathrm{~mm}$.

- Pixel resolution is defined as the number of gray levels that are represented in a pixel. It is also known as gray scale resolution. For the JAI CV M1 CCD camera, the pixel resolution is 8 bit.

\subsection{Calculation of CCD sensor size:}

CCD Sensor Size defined as the size of a camera sensor's active area, typically specified in the horizontal dimension. For $2 / 3$-inch format JAI CV M1 CCD Camera, the sensor size is 8.8 X $6.6 \mathrm{~mm}$.

\subsection{Calculation of Field of View:}

Field of View (FOV) is defined as the viewable area of the object under inspection or the portion of the object that fills the camera's sensor.

$$
\text { Field of view }=(\mathrm{Dp}+\mathrm{Lv})(1+\mathrm{Pa})
$$

where $\mathrm{Dp}$ is the required field of view, $\mathrm{Lv}$ is the maximum variation in part location and orientation and $\mathrm{Pa}$ is the allowance for camera pointing as a percentage. Then,

Field of view $=(30 \mathrm{~mm}+10 \mathrm{~mm})(1+10 \%)=44 \mathrm{~mm}$

The factor by which the FOV should be expanded depends on the skill of the people who maintain the vision system. A value of $10 \%$ is a common choice. In order to fill the monitor with the feature of interest more clearly, the FOV of $66 \mathrm{~mm}$ is selected.

\subsection{Focal Length:}

The focal length is a lens' pivotal parameter. To represent an object completely on the CCD chip, one should calculate the focal length for the object height and width.

Focal Length of the Width $=$

(WD* CCD Width) / (SOO+ CCD Width)

Focal Length of the Height $=$

(WD* CCD Height) / (SOO+ CCD Height)

where WD is Working Distance and SOO is Size Of Object. So,

Focal Length of Width $=$

$(1000 \mathrm{~mm} * 8.8 \mathrm{~mm}) /(30 \mathrm{~mm}+8.8 \mathrm{~mm})=226.8 \mathrm{~mm}$

To adjust focal lengths, Zoom Lens is necessary. Due to some disadvantages of Zoom lens such as weight, size and Price Fixed focal length lens whose focal length is lower than the calculated one can be used. Especially in the case of small objects, the working distance may be smaller than the selected lenses minimal working distance (MOD). In this case, extension rings is placed between the lens and the camera to decrease MOD.

\subsection{Depth of Field:}

It is defined as the maximum object depth needed in focus [3]. In other words, the difference between the closest and farthest distances an object may be shifted before an acceptable blur is observed is called depth of field (DOF).

$$
\begin{aligned}
& \mathrm{DOF}_{\text {NearLimit }}=\mathrm{WD} /\left\{1+\mathrm{Blurspot}^{*} \text { Iris }^{*}\left(\mathrm{WD}-\mathrm{FL} / \mathrm{FL}^{2}\right)\right\} \\
& \text { DOF Near Limit } \\
& =1000 /\left(1+0.005 * 1.4 *\left(1000-226.8 / 226.8^{2}\right)\right) \\
& =999.895 \mathrm{~mm} \text {. } \\
& \mathrm{DOF}_{\text {FarLimit }}=\mathrm{WD} /\left\{1+\mathrm{Blurspot} * \text { Iris } *\left(\mathrm{WD}-\mathrm{FL} / \mathrm{FL}^{2}\right)\right\} \\
& \text { DOF Far Limit } \\
& =1000 /\left(1-0.005 * 1.4 *\left(1000+687.5 / 687.5^{2}\right)\right) \\
& =1000.105 \mathrm{~mm} \text {. }
\end{aligned}
$$

\subsection{Exposure time:}

This is the time during which light is creating an exposure. To hold image blur to within one pixel, the exposure time must be,

$$
\mathrm{T}_{\mathrm{e}}=\mathrm{FOV} /\left(\mathrm{V}_{\mathrm{p}} * \mathrm{~N}_{\mathrm{p}}\right)
$$

where $T_{e}$ is the exposure time, $V_{p}$ is the velocity of the conveyor and $\mathrm{N}_{\mathrm{p}}$ is the number of pixels in horizontal direction. Then, $\mathrm{T}_{\mathrm{e}}=66 /(800 * 1300)=0.00006$ seconds 
This exposure can be achieved with electronic shutter or strobed illumination.

\subsection{Image blur:}

Blurred images are caused by poor lens quality and incorrect lens setup. In this application, since the bottles are traveling in the conveyor line, there will be image blur. The magnitude of the image blur depends on the rate of part motion, the size of FOV, and the exposure time. The expression for the magnitude of blur is given by,

$\mathrm{B}=\mathrm{V}_{\mathrm{p}} * \mathrm{~T}_{\mathrm{e}} * \mathrm{~N}_{\mathrm{p}} / \mathrm{FOV}$

where $\mathrm{B}$ is the blur in pixels. The actual blur for this application can then be calculated as

$\mathrm{B}=800 * 0.00006 * 1300 / 66=0.9454$ pixels.

\section{INDUSTRIAL APPLICATIONS}

The main concern of using machine is to recognize that the part is well made according to the specified qualifications. In this field, the most significant task of the machine is for the automated visual inspections [AVIs]. AVI contribute a significant role in the automation field. AVI considerably enhances the automation capability of manufacturing process. The automated vision system can be used for the purpose of measurements, gauging, integrity checking, and quality control. In the area of measurements and gauging, the gauging of small gaps, measurements of the object dimension, alignment of the components, and the analysis of crack formation are common applications. Integrity checking in automotive plants, food industry and other production lines is performed by using such a vision system.

The medical and pharmacological products can be inspected by the machine vision systems. Using such an inspection method in the production line has increased the speed and reliability of the inspections. For example, during the automotive assembly, a vision guided robot recognizes the orientation of the engine heads and picks and places them correctly on the engine blocks [11]. In another case, a system examines the fiber optics assembly line. A PC-based imaging system integrates hardware and software to analyze the captured images for the possible fiber blemishes, chips, and cracks [12]. As an another example, in aerospace industry, a vision-based robot using the self-calibrating and self-teaching techniques has been reported that punches rivets into the airplane metal sheets with the high accuracy [13].

\section{CONCLUSION}

In this paper, a machine vision system for inspecting bottles with the bottle neck of $30 \mathrm{~mm}$ diameter has been described. JAI CV M1 progressive scan monochrome high resolution 2/3" CCD camera can be utilized for this application. The Design parameters of the Machine Vision system for the above application are given below:

Image resolution
Spatial resolution
Pixel resolution
Sensor size
Field of view
Focal Length

$1300 \times 1030$ pixels
$0.0067 \times 0.0067 \mathrm{~mm}$
$8 \mathrm{bit}$
$8.8 \times 6.6 \mathrm{~mm}$
$66 \mathrm{~mm}$
$226.8 \mathrm{~mm}$

$\begin{array}{ll}\text { DOF }_{\text {Near Limit }} & 999.895 \quad \mathrm{~mm} \\ \text { DOF }_{\text {Far Limit }} & \mathbf{1 0 0 0 . 1 0 5 ~} \mathrm{mm} \\ \text { Exposure time } & \mathbf{0 . 0 0 0 0 6} \text { seconds } \\ \text { Image blur } & \mathbf{0 . 9 4 5 4} \text { pixels }\end{array}$

The proposed procedure can be used to design machine vision system for the inspection of any quality parameter of a product in the manufacturing line itself.

\section{REFERENCES}

[1] Kannan. K., Muruganantham. C., and Jawahar. N., May 24\&25, 2004, Radial Alignment Constraint Based CCD Camera Calibration using 2D high accuracy target, Challenges in achieving Global Quality, Thiagarajar College of Engineering, Madurai, Proceedings, pp 100107.

[2] Kannan. K., Suresh Babu. R., Gamishilbha. G. and Dhanalakshmi. S. V., Jan 7\&8, 2005, Geometric and Radiometric Model of Imaging System for Robotic Vision, Competitive Manufacturing Technology and Management for Global Marketing, Crescent Engineering College, Chennai, Proceedings, pp 49-54.

[3] Kannan. K., Karpagavalli. K. and Sivasankari. K., Mar 17\&18, 2005, Design of Imaging System for Machine Vision Applications, Recent Trends in Design and Manufacturing Technologies, Kumaraguru College of Technology, Coimbatore, Proceedings.

[4] A.R. Rao, Future directions in industrial machine vision: a case study of semiconductor manufacturing applications, Image and Vision Computing 14 (1996) 3-19.

[5] R.T. Chin, Automated visual inspection-1981 to 1987, Computer Vision Graphics and Image Processing 41 (1988) 346-381.

[6] O. Mohtadi, J. Sanz, Recent progress in industrial machine vision, International Journal of Robotics and Automation 8 (1993) 44-66.

[7] T.S. Newman, A.K. Jain, A survey of automated visual inspection, Computer Vision and Image Understanding 61 (1995) 231-262.

[8] A.D.H. Thomas, M.G. Rodd, J.D. Holt, C.J. Neill, Realtime industrial visual inspection: a review, Real-Time Imaging 1 (1995) 139-158.

[9] A.M. Wallace, Industrial applications of computer vision since 1982, IEE Proc. E Comp. Digital Tech. 135 (1988) 117-136.

[10] Awcock GJ, Thomas R. Applied image processing. London: Mac Millan New Press Ltd.; 1995.

[11] Spotlight on robotics, Vision System Design, May 2003.

[12] Spotlight, Vision System Design, July 2002.

[13] Robots \& Vision, Vision System Design, July 2003. 